\title{
A critical review of FEM models to simulate the nano-impact test on PVD coatings
}

\author{
Georgios Skordaris ${ }^{1,2 *}$, Konstantinos Bouzakis ${ }^{1,2}$, and Paschalis Charalampous ${ }^{1,2}$ \\ ${ }^{1}$ Laboratory for Machine Tools and Manufacturing Engineering, Mechanical Engineering \\ Department, Aristotle University of Thessaloniki, Greece \\ ${ }^{2}$ Fraunhofer Project Centre Coatings in Manufacturing, in LMTME and in Fraunhofer Institute for \\ Production Technology in Aachen, Germany
}

\begin{abstract}
Nano-impact test is a reliable method for assessing the brittleness of PVD coatings with mono- or multi-layer structures. For the analytical description of this test, a 3D-FEM Finite Element Method (FEM) model and an axis-symmetrical one were developed using the ANSYS LS-DYNA software. The axis-symmetrical FEM simulation of the nano-impact test can lead to a significantly reduced computational time compared to a 3D-FEM model and increased result's accuracy due to the denser finite element discretization network. In order to create an axissymmetrical model, it was necessary to replace the cube corner indenter by an equivalent conical one with axis-symmetrical geometry. Results obtained by the developed FEM models simulating the nano-impact test on PVD coatings with various structures were compared with experimental ones. Taking into account the sufficient convergence between them as well as the reduced calculation time only in the case of an axis-symmetrical model, the latter introduced numerical procedure can be effectively employed to monitor the effect of various coating structures on their brittleness.
\end{abstract}

\section{3D-FEM model to simulate the nano-impact test}

The nano-impact test is a reliable method to characterize the brittleness of PVD coatings $[1,2,3,4,5]$. Efforts for the numerical description of the nano-impact test have been undertaken in the past by developing a three dimensional (3D) FEM-model considering uniform or graded strength properties versus the coating thickness [2,3] (see figure 1). In these calculations, the simulation of the applied cube corner indenter geometry is in accordance to the manufacturer specifications. During the indenter penetration into the film, it is assumed that the coating at the FEM model node regions can withstand the applied load up to a maximum value, which corresponds to the coating rupture stress and the associated maximum plastic strain limit. Over these limits, the related nodes are disconnected from the neighboring finite elements. If all nodes of an element are disconnected, the element is released for simulating a crack formation and becomes an inactive separate entity. For minimizing the FEM calculations solving time, the nodes'

*Corresponding author: gskor@eng.auth.gr 


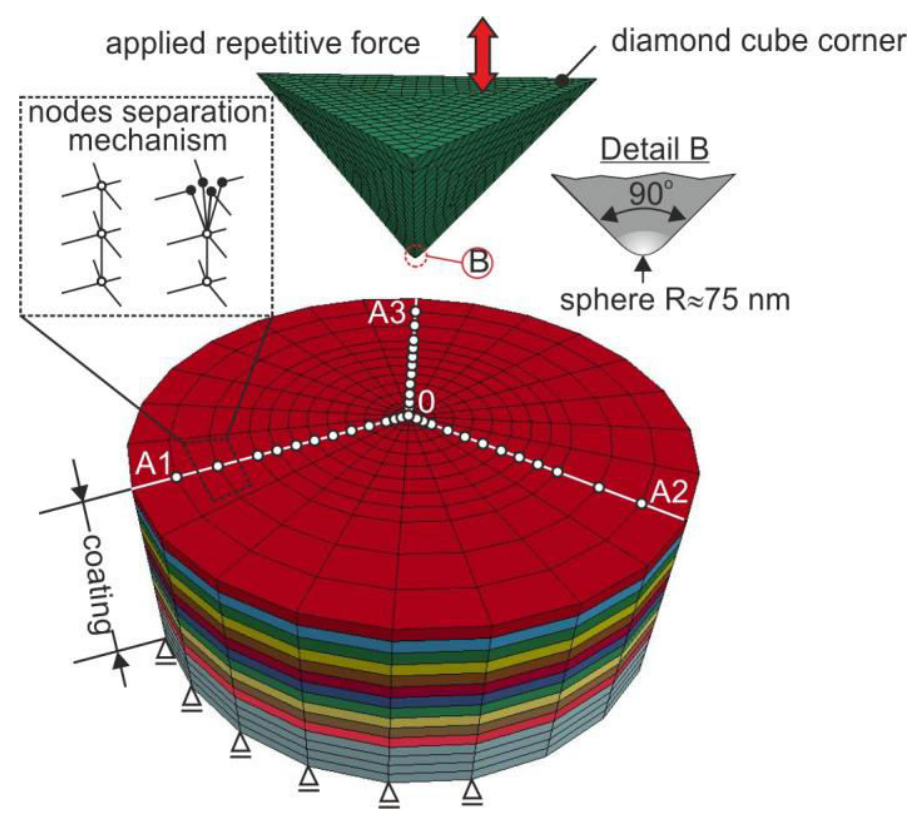

Fig. 1. The developed 3D-FEM model for simulating nano-impact test.

ability to be disconnected is restricted to those nodes, which are located on the perpendicular to the coating surface section levels OA1, OA2, OA3. The edges of the cube corner indenter lie on these levels during the indenter penetration into the film material. In this way, the stress fields developed in the coating and its fracture evolution in terms of
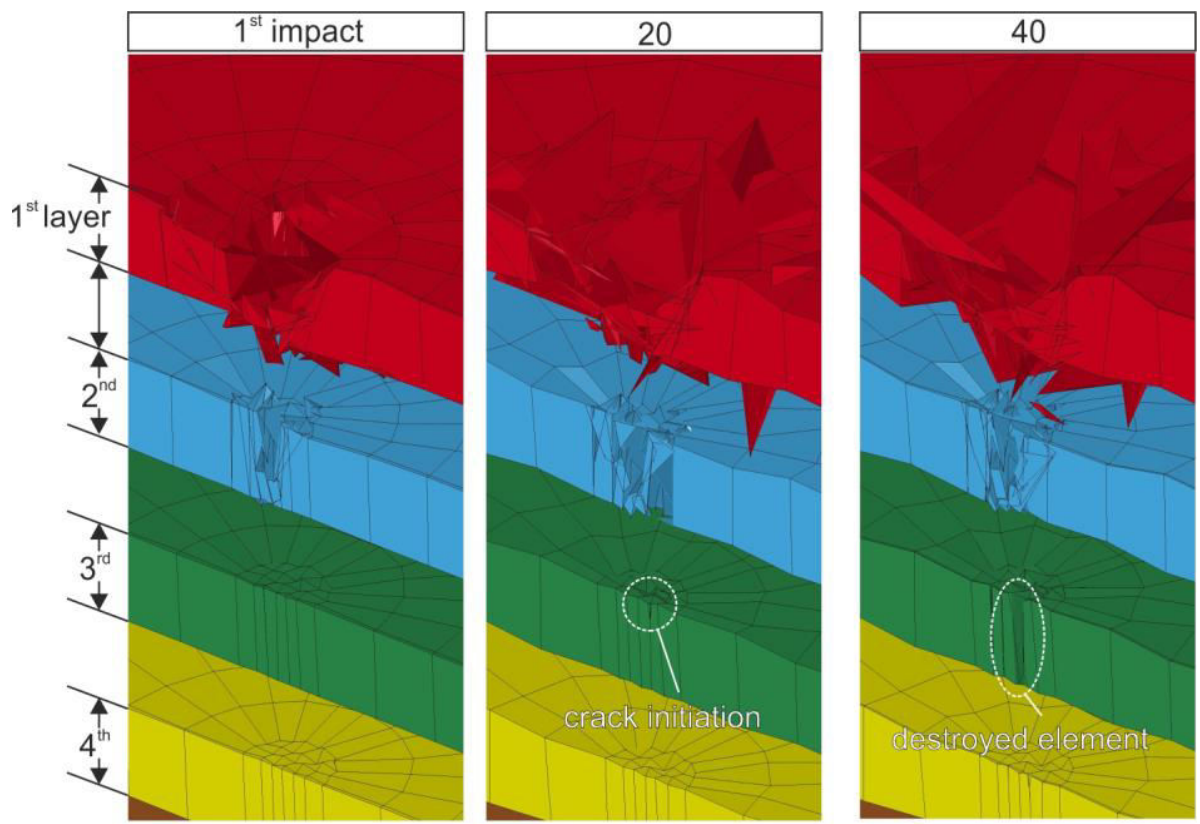

Substrate: $\mathrm{HW}-\mathrm{K} 05 / \mathrm{K} 20$, Coating: $\mathrm{Ti}_{46} \mathrm{Al}_{54} \mathrm{~N}, \mathrm{t} \approx 3.5 \mu \mathrm{m}$

Indenter : diamond cube, frequency : $1 \mathrm{~Hz}$, load : $30 \mathrm{mN}$

Fig. 2. 3D-depiction of the crack propagation during nano-impact test on PVD coatings. 


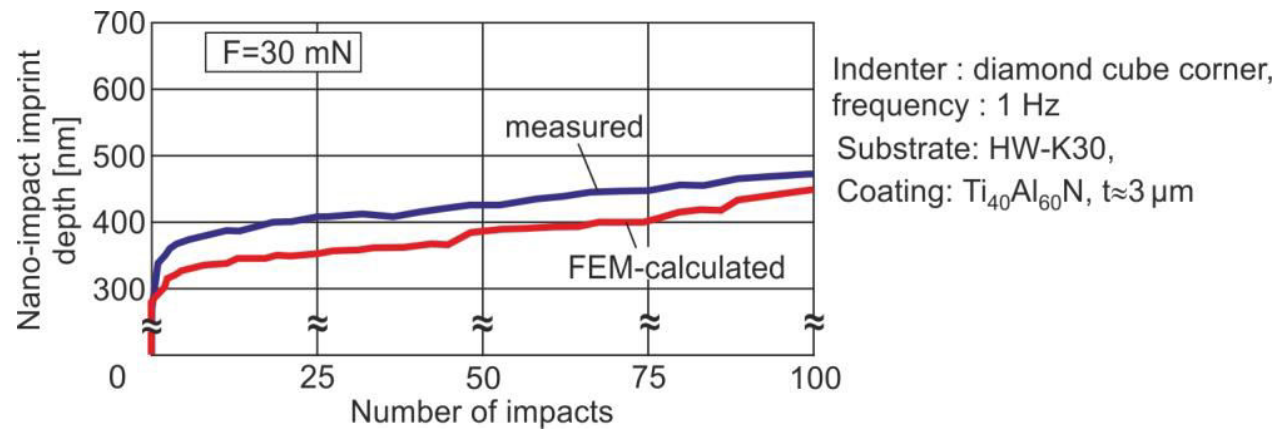

Fig. 3. Comparison between experimental and FEM-calculated imprint depths versus the number of impacts by using the 3D-FEM model.

imprint depth versus the repetitive impacts can be analytically described. A characteristic example, indicating the crack propagation and thus the impact depth versus the number of impacts is shown in figure 2. A correlation between measured and FEM calculated imprint depths after the conduct of nano-impact test on TiAlN coated inserts reveals a sufficient convergence as shown in figure 3. However, for attaining a reasonable accuracy by applying the 3D-FEM model, fine discretization networks have to be applied, leading to long calculation times. In this way, its application for a precise description of fine coating structures is limited.

\section{Axis-symmetrical FEM model to simulate the nano-impact test}

\subsection{The developed axis-symmetrical FEM model}

Since an axis-symmetrical FEM simulation of the nano-impact test can lead to a significantly reduced computational time compared to a 3D-FEM model [1], it was necessary to replace the cube corner indenter by an equivalent conical one with axissymmetrical geometry. The equivalent cone possesses the same projected area versus the indentation depth $h_{i}$ (see figure 4). The angle of the equivalent cone was calculated according to equations introduced in [6]. Hereupon, the actual spherical tip radius of the cube corner indenter equal to roughly $75 \mathrm{~nm}$ was taken into account.

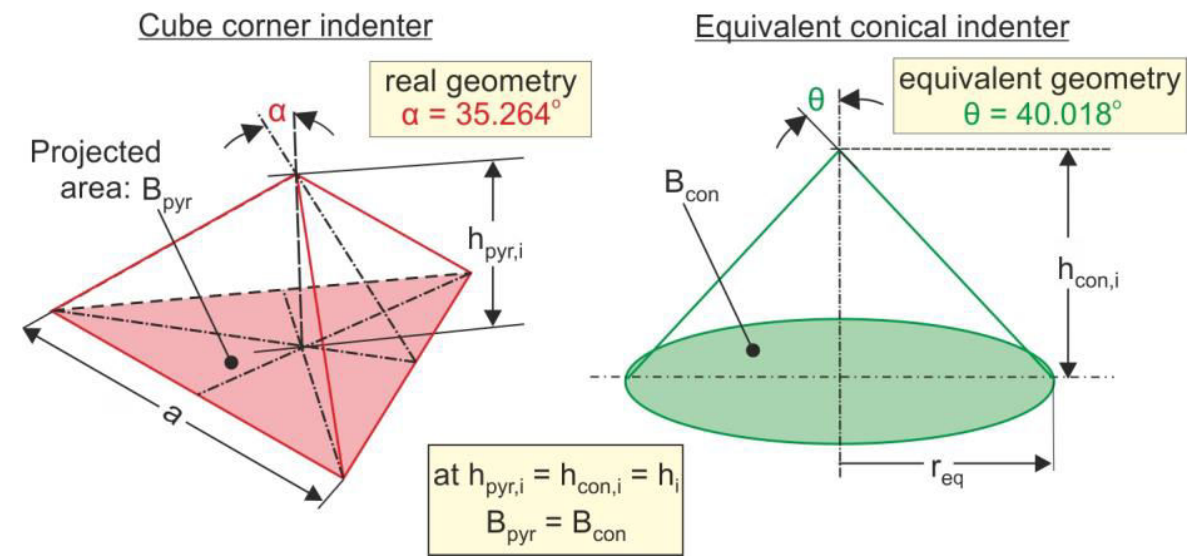

Fig. 4. Description of a diamond cube corner indenter by means of an equivalent cone. 


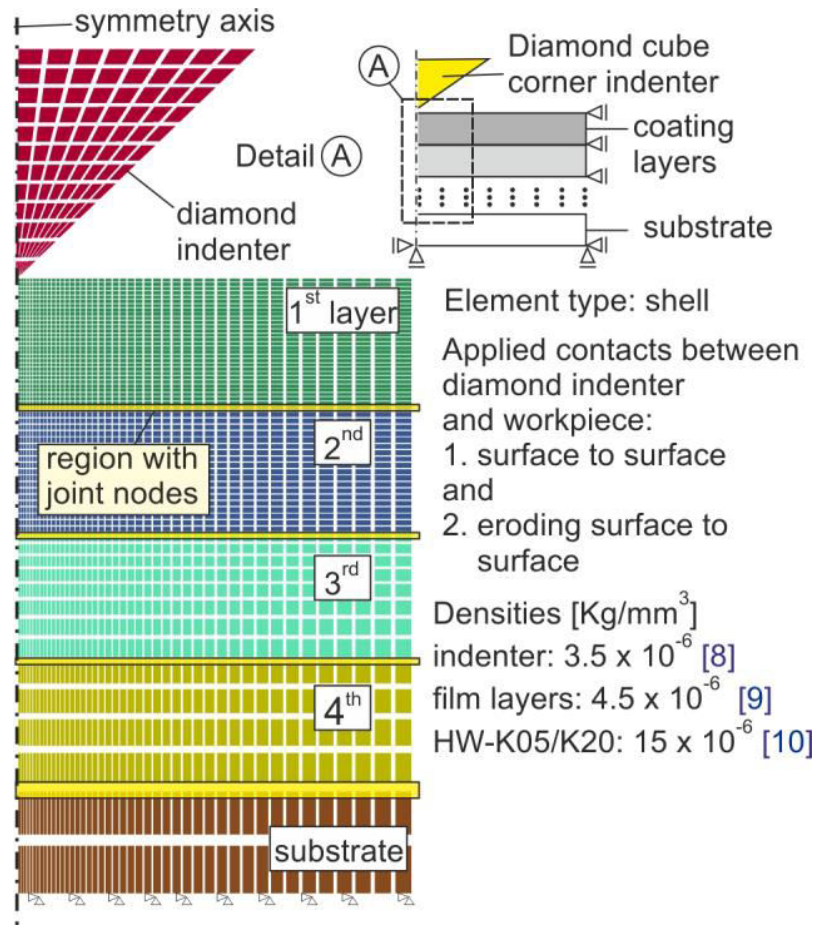

Fig. 5. The developed axis-symmetrical FEM model for simulating the nano-impact test using the LSDYNA software.

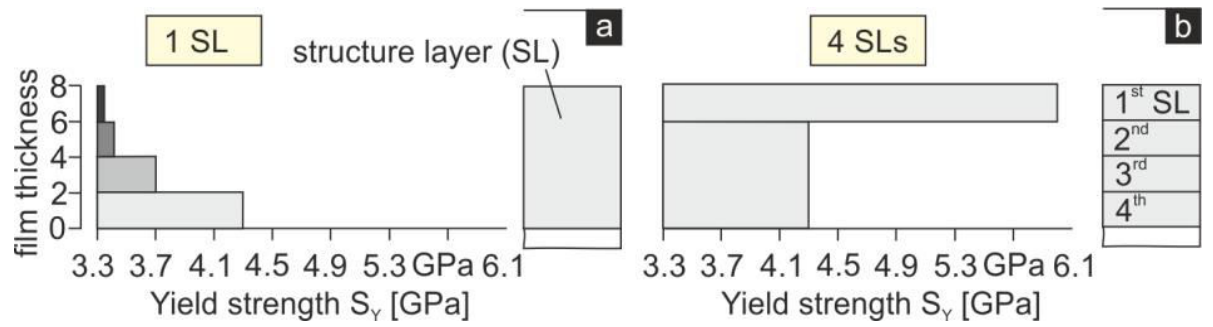

Fig. 6. Predicted graded strength properties of $8 \mu \mathrm{m}$ thick coatings with different structures.

An axis-symmetrical FEM model was created, using the ANSYS LS-DYNA software package. The developed FEM model consisting of individual shell elements is demonstrated in figure 5. The applied element formulation option is characterized in LSDYNA as axis-symmetrical solid-area weighted [7]. The actual film strength properties were estimated taking into account the coating structure shown in figure 6 . In these calculations, materials with piecewise linear plasticity and strain rate independent were considered. It was reasonable to assume that the film strain rate does not affect the developed film strains, since the duration of the nano-impact test lasts $1 \mathrm{~s}$ and strains of the applied film material are affected by the strain rate at impact force durations less than few milliseconds [8]. The diamond indenter was assumed as rigid. Calculations were also conducted for an elastic diamond indenter. In both cases, the obtained results were practically identical. Because the calculation time is comparably shorter in the case of a rigid indenter, this option was employed. The densities of the involved materials in the FEM model are documented in $[9,10,11]$. For describing the indenter penetration into the coating material, it was assumed that the coating layers and the substrate behave as 


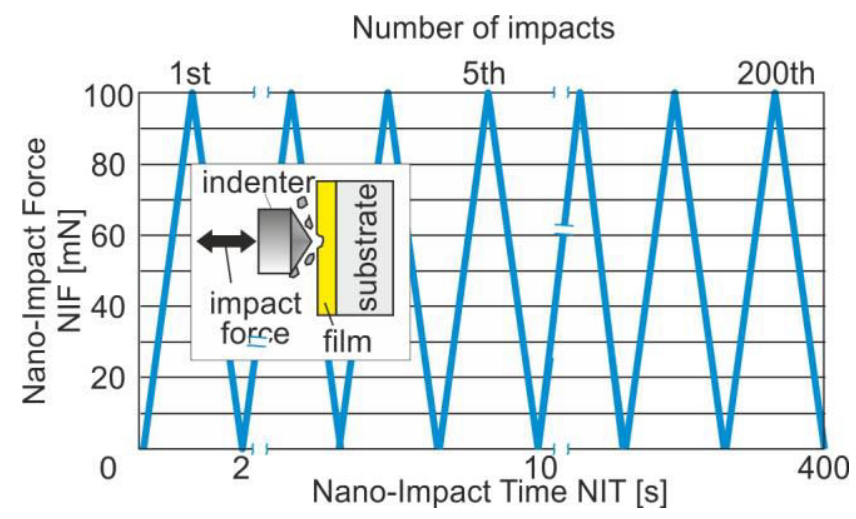

Fig. 7. Measured time course of the force during the nano-impact test, considered in the FEMcalculations.

individual bodies with own strength properties. Moreover, nodes belonging to neighborhood elements between two coating layers were joined in one.

In the developed FEM model, a surface to surface contact was applied for describing the interface between the diamond indenter and the coated specimen. This is a penalty-based contact with springs placed between all penetrating nodes and the contact surface [7,12]. In addition, an eroding contact, also a penalty-based contact, was applied between each individual coating layer and the indenter [6]. In this way, elements involved in the contact definition are subject to erosion (element deletion) according to a material failure criterion and not directly due to the eroding contact restrictions. The contact surface is updated as external elements are deleted. In the performed calculations, it was assumed that each coating layer can withstand the applied load up to a maximum value correspondingg to its rupture strain and rupture stress. If the developed element strain exceeds the rupture strain, then the element is deleted for simulating the crack and debris formation. The accuracy of discretizising the coating thickness could be more than twenty times higher than that of the developed in the past related FEM model $[2,3]$, thus attaining a more detailed description of the coating structure and its damage. Moreover, due to the axis-symmetrical FEM model structure, the FEM calculation solving time is comparably significantly shorter.

For attaining the diamond indenter motion, a concentrated nodal force on the indenter mass center is applied [7]. The time course of this load is linked to a certain curve representing the time dependent impact force, as it is shown in figure 7 . The equilibrium differential equations are integrated for incremental solution time steps of few milliseconds. Each solution step is based on the results of the previous one (explicit method).

\subsection{Characteristic results obtained by the developed FEM model}

The impact depths and equivalent stress fields after the first, one hundred and two hundred impacts in various coating structure cases are exhibited in figure 8 . These results are associated with coatings possessing one or four structure layers (SLs). The corresponding to the coating's structures properties are documented in figure 6 . As the number of the impact cycles increases, the indentation depth grows as well. Due to the lower mechanical properties and higher brittleness of the mono-layer coating compared to the four-layered one, the related nano-impact depths are larger.

Comparisons between measured and FEM calculated imprint depths versus the number of impacts, for two coatings both of $8 \mu \mathrm{m}$ thickness but with one or four SLs are illustrated in figure 9. The FEM calculated imprint depths converge sufficiently with the measured 

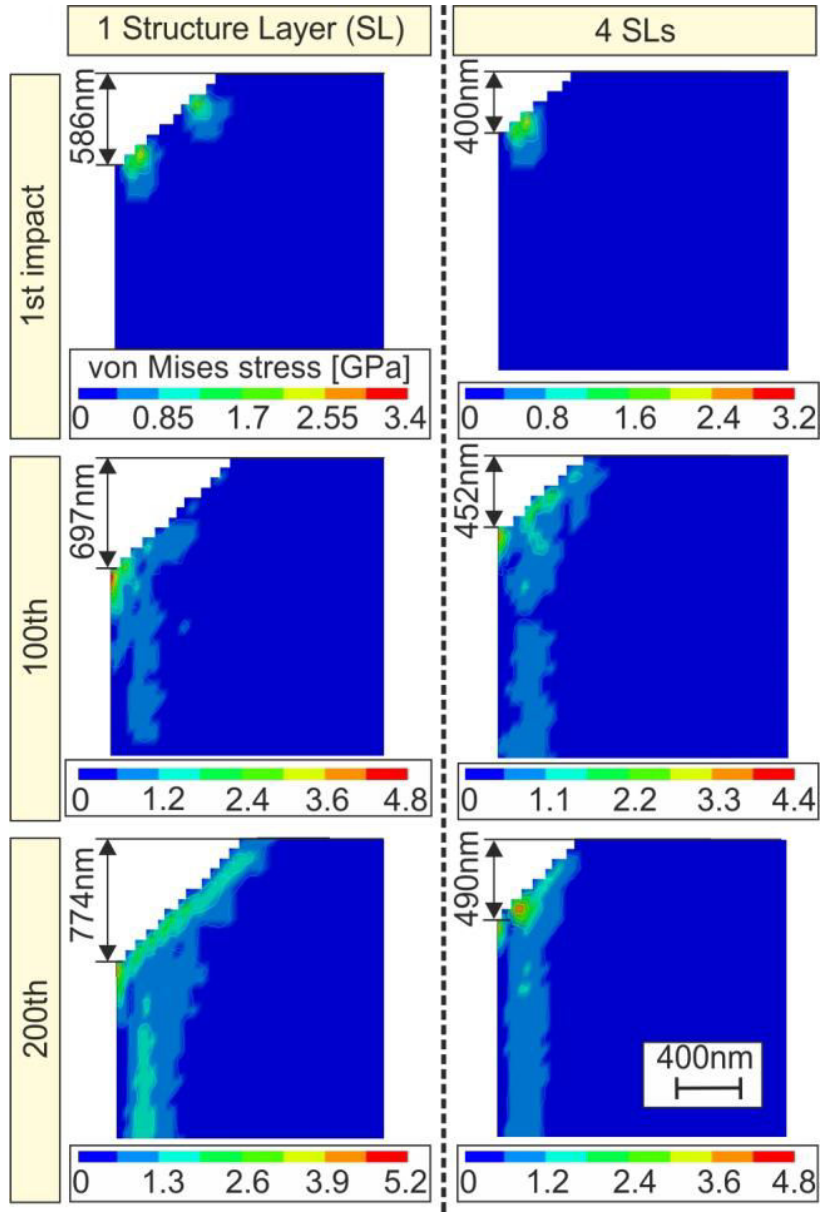

Fig. 8. Developed equivalent stress fields after various impact numbers in the case of a $8 \mu \mathrm{m}$ thick PVD coating with 1 or 4 SLs, employing a diamond cube indenter at a frequency of $1 \mathrm{~Hz}$ and a maximum load of $100 \mathrm{mN}$.

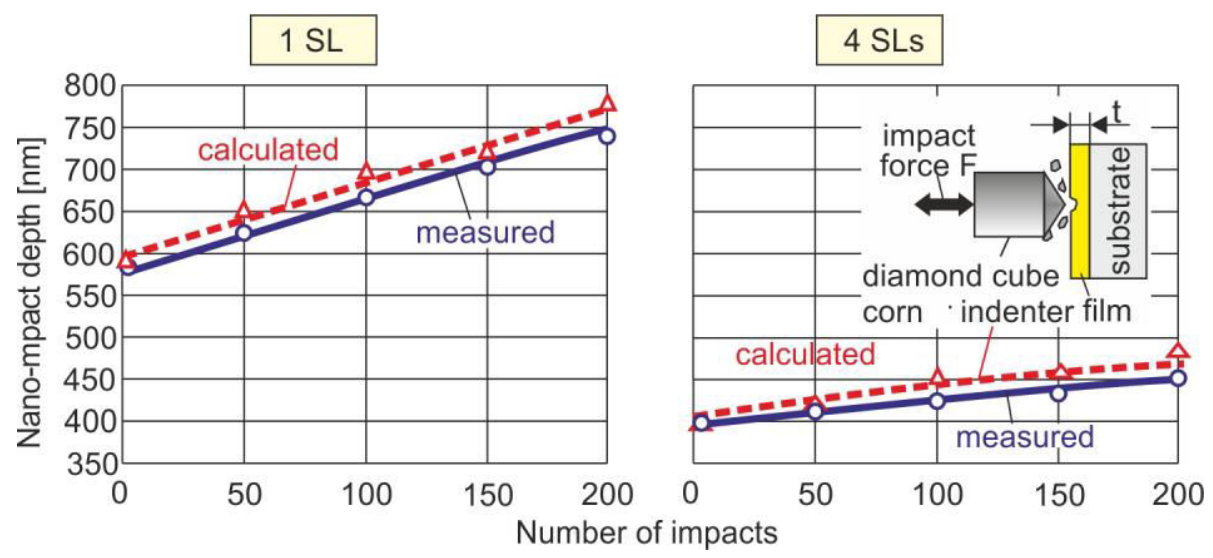

Fig. 9. Comparison between experimental and FEM-calculated imprint depths versus the number of impacts on variously structured coatings, considering graded strength properties versus the film thickness (diamond cube indenter at a frequency of $1 \mathrm{~Hz}$ and a maximum load of $100 \mathrm{mN}$ ). 
ones, if the existing strength property gradations are considered. The corresponding deviations from the measured results are less than 5\%. In this way, the developed FEM model can be effectively applied for assessing the brittleness of PVD coatings with various structures and graded strength properties. Moreover, the obtained experimental and analytical results ascertain the fact that coatings with multi-layer structures, due to their low brittleness, can withstand more effectively impact loads compared to mono-layer ones. In this context, the presented FEM model can be employed to evaluate the efficiency of potential mechanical or thermal coating treatments for reducing the coating brittleness.

\section{Conclusions}

The nano-impact test is a reliable method for evaluating the coating brittleness. This test was dynamically simulated by developing a 3D-FEM model and an axis-symmetrical FEM one. In these calculations, PVD coatings with various structures were employed. By the introduced axis-symmetrical FEM simulation, compared to the 3D-FEM one, the calculation's time was significantly reduced and moreover, the result's accuracy can be increased due to the denser finite element discretization network. In this way, the axissymmetrical FEM model could be an efficient tool for assessing film structure's and mechanical properties gradation's effects on the coating's brittleness.

\section{References}

1. G. Skordaris, K.-D. Bouzakis, P. Charalampous, Surf. Coat. Technol. 265, 53 (2015)

2. K.-D. Bouzakis, G. Skordaris, S. Gerardis, E. Bouzakis, Materialwissenschaft und Werkstofftechnik 44 (8), 684 (2013)

3. K.-D. Bouzakis, S. Gerardis, G. Skordaris, E. Bouzakis, Surf. Coat. Technol. 206, 1936 (2011)

4. B.D. Beake, G.S. Fox-Rabinovich, S.C. Veldhuis, S.R. Goodes, Surf. Coat. Technol. 203, 1919 (2009).

5. B.D. Beake, G.S. Fox-Rabinovich, Surf. Coat. Technol. 255,102 (2014).

6. K.-D. Bouzakis, M. Pappa, G. Maliaris, N. Michailidis, Surf. Coat. Technol. 215, 218 (2013)

7. LS-DYNA Keyword user's manual, Version 971, 2007. (Livermore Software Technology Corp.).

8. K.-D. Bouzakis, G. Maliaris, S. Makrimallakis, Int. J. Fatigue 44, 89 (2012)

9. J.E. Graebner, Diam. Relat. Mater. 5,1366 (1996)

10. Inge L. Rasmussen, Optical Monitoring and X-Ray Absorption Spectroscopy for Studies of Wear on Thin Films, PhD thesis, University of Copenhagen, Denmark, 2011

11. ISO 3369:2006 (standard EN 23 369) Impermeable sintered metal materials and hardmetals-Determination of density.

12. G. Skordaris, J. Mater. Eng. Perform. 22, 3192 (2013) 